\title{
„Alle wahren Gefühle verbergen und mit fester Stimme und wohlformulierten Sätzen glänzen!"- Die Bedeutung von Selbstdarstellungsregeln im Bewerbungsinterview
}

\author{
Monika Sieverding
}

\begin{abstract}
Zusammenfassung. Im Labor wurde eine Bewerbungssituation simuliert, in der 74 Männer und Frauen individuell einen schriftlichen Leistungstest, einen Vortrag zur Selbstdarstellung der beruflichen Qualifikation sowie ein standardisiertes Bewerbungsinterview absolvierten. Vortrag und Bewerbungsinterview wurden videografiert. Die Länge des Vortrags zur Selbstdarstellung (maximal fünf Minuten) und der nonverbale Gefühlsausdruck im Gesicht dienten als unabhängige Variablen; abhängige Variablen waren Selbst- und Fremdeinschätzungen als ,,erfolgreich“. Entsprechend den Hypothesen wurden solche Bewerber als erfolgreicher eingeschätzt, die in der Selbstdarstellung länger geredet hatten und die ein neutrales Gesicht zeigten.

Schlüsselwörter: Bewerbungsinterview, Selbstdarstellung, Gefühlsausdruck
\end{abstract}

The role of display rules in a job interview situation

\begin{abstract}
A job interview situation was simulated in the laboratory. Each of the 74 men and women who participated was asked to complete a written test, a verbal self-presentation of his or her professional qualifications, and a personal interview. The verbal selfpresentation and the personal interview were videotaped. The duration of the verbal self-presentation (maximum five minutes) and the facial expressiveness served as independent variables, the self-evaluations and external assessments as „successful" were the dependent variables. In accordance with the hypotheses, subjects who talked longer in the self-presentation speech and subjects who showed a neutral face in the personal interview were rated as more successful in mastering the job interview.

Key words: Job interview, diplay rules, self-presentation, expressiveness
\end{abstract}

\section{Fragestellung}

Im Prozess der psychologischen Personalauswahl sind Auswahl- oder Einstellungsgespräche nach der Auswertung der schriftlichen Bewerbungsunterlagen die verbreitetste Methode. Dabei entsteht das Gesamturteil häufig intuitiv (Schuler, 1996), und die entscheidenden Einflussgrößen auf die individuelle Urteilsbildung der beteiligten Beobachter sind noch weitgehend unbekannt (Graudenz, 1995).

Eine Bewerbungssituation ist eine belastende Leistungssituation, deren Ausgang für den Bewerber oft mit lebenswichtigen Veränderungen und Konsequenzen verbunden ist. Graudenz (1987, zit. in Jäger, 1995) fand, dass ca. zwei Drittel der Bewerber Gefühle von Unsicherheit, Anspannung und Unruhe äußerten; die Hälfte der Befragten berichtete von leichten psychosomatischen Störungen. Es scheint jedoch wenig ratsam, sich diese Gefühle und den durch die Situation erzeugten Stress anmerken zu lassen. Von Seiten der Einstellenden wird nämlich erwartet, dass ein Bewerber Sicherheit im

Ich danke Dr. Bettina von Volkmann für die Versuchsleitung sowie Dr. Birgit Herbeck für die Durchführung des Ratertrainings. nonverbalen Verhalten demonstriert und sich im Gespräch als aktiver und gleichberechtigter Gesprächspartner zeigt (Jochmann, 1984; Wottawa, 1995). Entsprechend empfehlen professionelle Ratgeber für ein erfolgreiches Abschneiden den Einsatz von „schauspielerischen Fähigkeiten“, um die „strahlende Seite“ der eigenen Persönlichkeit vorzuführen (z. B. Hesse \& Schrader, 1994). Ekman und Friesen $(1969,1978)$ postulierten die Existenz von Display-Rules (Darbietungs- oder Darstellungsregeln), in denen festgelegt ist, wer wem gegenüber wann welches Gefühl zeigen bzw. nicht zeigen sollte. Situativ wirksame Darstellungsregeln können den Gefühlsausdruck intensivieren (z.B. Freude über ein Geschenk oder Trauer bei einer Beerdigung zeigen müssen), aber auch eine Hemmung der Expressivität verursachen („Poker-Face“, s. Bänninger-Huber \& Salisch, 1994). Die vielfach beobachtete Verhaltenstendenz zur strategischen und positiven Selbstdarstellung (Köchling \& Körner, 1996) verweist auf die Wirkung von DisplayRules in Bewerbungssituationen; auch die Befunde von Diemand und Schuler (1991) sprechen dafür. Sie fanden, dass assertive Selbstdarstellung, die Fähigkeit, „sich gut verkaufen zu können“, der wesentliche Prädiktor für das erfolgreiche Abschneiden in einem Assessment-Center war. Allerdings wurde in ihrer Studie die 
Fähigkeit zur assertiven Selbstdarstellung über einen Fragebogen - eine Self-Monitoring-Kurzskala - erfasst und nicht über das tatsächliche Verhalten der Bewerber im Assessment-Center.

Selbstsicherheit kann über verschiedene Kanäle ausgedrückt werden: Neben dem visuellen Ausdrucksverhalten (Gestik, Mimik, Körperhaltung) sind vor allem auditive Aspekte wichtig, wie die Stimmqualität oder die Sprechweise (Wallbott, 1995). Auch wenn die Kürze bzw. Prägnanz einer Mitteilung als ,kommunikative Kompetenz" angesehen wird (Fittkau, 1995), gilt die Sprachaktivität bzw. Sprechdauer als Indikator für Selbstsicherheit (Jochmann, 1984; Wottawa, 1995). In den meisten Vorstellungsgesprächen wird den Bewerbern die Gelegenheit gegeben, sich selbst vorzustellen. Hier geht es darum, eine aktive und selbstbewusste Darstellung der eigenen Person zu liefern. Meine Vermutung war, dass - unabhängig von den Inhalten - die Sprechzeit, d.h. die Dauer einer solchen Selbstdarstellung, sowie die Expressivität des Gesichtes das Urteil über den Bewerber beeinflussen. Folgende Thesen sollten überprüft werden:

H1: Ein Bewerber, der die vorgegebene Zeit zur Selbstdarstellung nutzt, wird erfolgreicher eingeschätzt als ein Bewerber, der sich nur kurz darstellt und die vorgegebene Zeit nicht ausnutzt.

H2: Je neutraler das Gesicht eines Bewerbers ist, d.h., je weniger Gefühle (von Angst oder Unsicherheit) und Anspannung zu sehen sind, desto erfolgreicher wird er oder sie eingeschätzt.

\section{Methode}

\section{Vorgehensweise}

Es wurde im Labor eine Bewerbungssituation simuliert, in der 74 Männer und Frauen individuell einen schriftlichen Leistungstest, einen Vortrag zur Selbstdarstellung der beruflichen Qualifikation sowie ein standardisiertes Bewerbungsinterview absolvierten. Eine Versuchsleiterin bediente die Geräte; die Instruktionen erfolgten über ein Tonbandgerät. Bei dem Vortrag zur beruflichen Selbstdarstellung hatten die Probanden die Aufgabe, in einem freien Vortrag die eigene Qualifikation für die von ihnen angestrebte Stelle darzustellen. In der Instruktion wurde ihnen mitgeteilt, dass sie für diesen Vortrag fünf Minuten Zeit hätten und dass sie die zur Verfügung stehende Zeit so gut wie möglich mit ihrer Selbstdarstellung füllen sollten. Bei dem Bewerbungsinterview wurden Fragen von einem männlichen Interviewer über Tonkassette eingespielt, um die Versuchsbedingungen des Interviews zu standardisieren und Interaktionseffekte in der sozialen Eindrucksbildung (Schuler, 1996) aus- zuschalten. Die Probanden hatten jeweils eine Minute Zeit, darauf zu antworten. Es wurden zehn Fragen gestellt, wie sie typisch für Bewerbungsinterviews, insbesondere für Stressinterviews, sind (z.B. „Was qualifiziert Sie eigentlich für diese Stelle?“ „Wozu sind Sie sich zu schade?"). Den Probanden wurde gesagt, dass ihre Leistungen später von Personalexperten beurteilt würden.

\section{Probanden}

Die Stichprobe $(N=74)$ bestand aus 37 Männern und 37 Frauen aus verschiedenen Studienfächern der Freien Universität Berlin, für die eine berufliche Bewerbung in unmittelbarer oder absehbarer Zeit aktuell war. Die meisten Probanden befanden sich im Hauptstudium, kurz vor Abschluss des Studiums, einige hatten ihr Studium bereits abgeschlossen (4 Männer, 7 Frauen). Die Teilnehmer waren zwischen 22 und 34 Jahre alt; das Durchschnittsalter lag bei 26 Jahren $(M=26.4, S D=2.7)$.

\section{Variablen}

Dauer der Selbstdarstellung. Anhand der Videoaufzeichnung wurde die Dauer des Vortrags zur beruflichen Selbstdarstellung für jeden Probanden sekundengenau ermittelt.

Selbsteinschätzungsdaten. Die Probanden sollten nach jeder Aufgabe auf einer 10-stufigen Skala (von 0 „überhaupt nicht" bis 9 ,sehr stark“) einschätzen, wie erfolgreich sie sich in der vorangegegangen Untersuchungsphase gefühlt hatten. In einer Nachbefragung, die sich unmittelbar an die Untersuchung anschloss, wurden die Probanden - angeregt durch die Arbeiten von Hochschild (1979) und Weber (s. z. B. Weber \& Leger, 1993) - nach verhaltensleitenden Gefühls- und Verhaltensregeln gefragt.

Fremdeinschätzungsdaten. Die Expressivität des Gesichts wurde anhand eines Videoausschnitts aus dem Bewerbungsinterview (Beantwortung der Frage „Welche Schwächen haben Sie?") eingeschätzt. Eine Diplom-Psychologin / Betriebswirtin und eine Ärztin, die die Fragestellung und Hypothesen der Untersuchung nicht kannten, beurteilten bei allen Probanden die nonverbale Expressivität des Gesichts anhand vorgegebener Kategorien auf einer 10-stufigen Skala von 0 bis 9 (in Anlehnung an Herbeck, 1994, und Traue, 1989). Die Operationalisierung der Variable „Neutrales Gesicht" lautete folgendermaßen: „Im Gesicht sind keine Nervosität, Anspannung oder Ängstlichkeit zu erkennen; weder negative noch positive Emotionen sind erkennbar: man kann im Gesicht nicht ablesen, was der Proband emotional empfindet; ähnlich wie Nachrichtensprecher/ 
in." Die Inter-Rater-Übereinstimmung lag nach einem intensiven Rater-Training bei diesen Kategorien zwischen $\mathrm{r}=.8$ und $\mathrm{r}=.9$ (Rangkorrelationen). Im Anschluss an die Einschätzung der nonverbalen Expressivität wurden die Antworten der Probanden nach stimmlichen und inhaltlichen Kriterien ausgewertet. AbschlieBend wurde jeder Proband auf einer 10-stufigen Skala (von 0 ,überhaupt nicht“ bis 9 ,sehr stark“) danach eingeschätzt, wie erfolgreich er oder sie auf die Raterinnen gewirkt hat („Der Proband wirkt ...“). Die Urteile der Raterinnen wurden für jeden Probanden gemittelt.

\section{Ergebnisse}

\section{Selbstdarstellungsregeln}

In der Nachbefragung gaben die meisten Probanden an, dass ihr Verhalten während der Untersuchung, insbesondere während Selbstdarstellung und Bewerbungsinterview, durch Gefühls- und Verhaltensregeln beeinflusst wurde. Die Einzelnennungen lassen sich zu einer Regel zusammenfassen: Man sollte in einer Bewerbungssituation versuchen, körperliche Erregung wie Nervosität und Anspannung sowie Gefühle von Unsicherheit und Angst zu verbergen und stattdessen Selbstsicherheit demonstrieren. Einige Beispiele aus den Antworten sind in Tabelle 1 dargestellt.

Tabelle 1. Selbstdarstellungsregeln im Bewerbungsinterview

\footnotetext{
„Nicht unsicher wirken!“

„Keine Aufregung zeigen, nicht ängstlich sein!“

„Gefühle (Unsicherheit) beherrschen!““

„Zeige Gelassenheit, auch wenn Du innerlich erregt bist!"

„Sei cool!‘

„Versuchen, Sicherheit auszustrahlen!“

„Sich selbstbewusst darstellen!“”

„Möglichst sicher wirken, keine Schwächen zeigen!“

„Alle wahren Gefühle verbergen und mit fester Stimme und wohlformulierten Sätzen glänzen!“”
}

Anmerkungen. Angaben der Probanden in der Nachbefragung (Beispiele)

\section{Werden Bewerber, die sich entsprechend den Regeln verhalten, als erfolgreicher eingeschätzt?}

Die entsprechenden Hypothesen lauteten, dass Probanden, a) deren Vortrag zur Selbstdarstellung länger dauert und b) deren Gesicht überdurchschnittlich neutral ist (d.h. denen man keine Nervosität, Anspannung oder Ängstlichkeit ansieht), als erfolgreicher eingeschätzt werden im Vergleich zu Probanden, deren Selbstdarstellung nur kurz ist und die ein weniger neutrales Gesicht zeigen.

Dauer der Selbstdarstellung: Nur jeder vierte Proband $(n=18)$ nutzte die vorgegebene Zeit entsprechend der Instruktion voll aus. Das Spektrum reichte von 50 bis 300 Sekunden, der arithmetische Mittelwert lag bei $M=196$ Sekunden $(S D=84.1)$. Männer redeten im Durchschnitt fast eine Minute länger als Frauen $(M=$ 222.4, $S D=69.1$ versus $M=170.4, S D=90.1), t(71)=$ $2.76, p=.007$.

Neutrales Gesicht: Die Probanden zeigten im Bewerbungsinterview ein überdurchschnittlich neutrales Gesicht, der Mittelwert lag bei $M=6.0$ ( $S D=1.6)$, es gab keinen signifikanten Geschlechtsunterschied. In Tabelle 2 sind die Interkorrelationen zwischen den Untersuchungsvariablen dargestellt.

Die erste Hypothese wird durch die Daten voll bestätigt. Die Dauer der Selbstdarstellung ist positiv mit der Selbsteinschätzung als erfolgreich assoziiert, und zwar sowohl für die Aufgabe Selbstdarstellung $(r=.60, p<$ .01) als auch für die anschließende Aufgabe Bewerbungsinterview $(r=.37, p<.01)$. Auch die Fremdeinschätzung der Bewerber im Bewerbungsinterview ist positiv mit der Dauer der Selbstdarstellung korreliert $(r$ $=.44, p<.01)$ : Je länger ein Proband in der Aufgabenphase Vortrag zur Selbstdarstellung über sich selbst gesprochen hatte, umso erfolgreicher wurde er oder sie anschließend von den Raterinnen im Bewerbungsinterview beurteilt. Die zweite Hypothese konnte nicht im Hinblick auf die Selbsteinschätzung, wohl aber im Hinblick auf die Fremdeinschätzung bestätigt werden. Der Zusammenhang zwischen Neutralität und Selbsteinschätzung als erfolgreich im Bewerbungsinterview ist zwar in der erwarteten Richtung, jedoch nicht signifikant ( $r=$ .20); ein positiver signifikanter Zusammenhang besteht zwischen der Neutralität des Gesichtes und der Fremdeinschätzung als erfolgreich $(r=.25, p<.05)$. Je weniger im Gesicht eines Bewerbers abzulesen war, was er oder sie emotional empfindet, desto erfolgreicher wurde er oder sie von den Raterinnen eingeschätzt. Zwischen der Selbst- und Fremdeinschätzung als erfolgreich im Bewerbungsinterview besteht ein signifikanter Zusammenhang $(r=.49, p<.01)$.

Um abzuschätzen, welches Gewicht den Variablen Neutralität des Gesichtes im Bewerbungsinterview und Dauer der Selbstdarstellung zur Vorhersage der Fremdeinschätzungen als erfolgreich zukommt, wurde abschließend eine Regressionsanalyse (schrittweise Methode) gerechnet, in der das biologische Geschlecht als dritte Variable berücksichtigt wurde. Die Ergebnisse sind in Tabelle 3 dargestellt.

Die Variable Geschlecht ist kein signifikanter Prädiktor zur Vorhersage der Fremdeinschätzung als erfolg- 
Tabelle 2. Interkorrelationen zwischen den Untersuchungsvariablen

\begin{tabular}{|c|c|c|c|c|}
\hline & Dauer SD & $\begin{array}{l}\text { neutr. Ges. } \\
\text { (BI) }\end{array}$ & $\begin{array}{l}\text { S.-,,erfolgr.“ } \\
\text { (SD) }\end{array}$ & $\begin{array}{c}\text { S.-,,erfolgr.“ F.- ,erfolgr.“ } \\
\text { (BI) }\end{array}$ \\
\hline \multicolumn{5}{|l|}{ Dauer SD } \\
\hline Neutr. Ges. (BI) & .05 & & & \\
\hline S.-,,erfolgr.“(SD) & $.60 * *$ & .13 & & \\
\hline S.-,,erfolgr.“(BI) & $.37 * *$ & .20 & $.64 * *$ & \\
\hline F.-,,erfolgr.“ (BI) & $.44 * *$ & $.25^{*}$ & $.36 * *$ & $.49 * *$ \\
\hline
\end{tabular}

Anmerkungen : $N=74 ; \mathrm{S}$. = Selbsteinschätzung, F. = Fremdeinschätzung, $\mathrm{SD}=$ Selbstdarstellung, $\mathrm{BI}=\mathrm{Bewerbungs-}$ interview; Produkt-Moment-Korrelationen: * $p<.05, * * p<.01$.

reich. Um von den Raterinnen als erfolgreich eingeschätzt zu werden, musste eine Person bei der Selbstdarstellung möglichst lange geredet und im Bewerbungsinterview ein möglichst neutrales Gesicht gezeigt haben. Die beiden Variablen Länge der Selbstdarstellung und Neutrales Gesicht können 23\% der Gesamtvarianz des Kriteriums Fremdeinschätzung als erfolgreich aufklären (s. Tabelle 3).

Tabelle 3. Multiple Regressionsanalyse zur Vorhersage der Fremdeinschätzung als erfolgreich im Bewerbungsinterview

\begin{tabular}{lccc}
\hline Variable & Beta & $t$ & $p<$ \\
\hline Geschlecht & & $<1$ & n.s. \\
Dauer der Selbstdarstellung & .43 & 4.2 & .001 \\
Neutralität des Gesichts & .23 & 2.2 & .030 \\
\hline
\end{tabular}

Anmerkungen: Schrittweise Methode; $N=74 . R^{2}=.25, R_{\text {adj. }}^{2}=.23$.

\section{Diskussion}

Das Anliegen der vorliegenden Studie war es herauszufinden, inwiefern die selbstsichere Selbstdarstellung eines Bewerbungskandidaten seinen oder ihren Erfolg im Bewerbungsinterview beeinflusst, und zwar unabhängig von fachlichen Qualifikationen. Es konnte gezeigt werden, dass Bewerber, die die vorgegebene Zeit zur Selbstdarstellung nutzten und möglichst lange redeten, sowie Bewerber, denen es gelungen ist, sich im Bewerbungsinterview keine Nervosität, Anspannung oder Ängstlichkeit anmerken zu lassen, als erfolgreicher eingeschätzt wurden im Vergleich zu Bewerbern, die die vorgegebene Zeit nicht nutzten und denen man Gefühle und Anspannung besser ansehen konnte. Dabei ist zu bedenken, dass die Raterinnen einen Ausschnitt aus dem Bewerbungsinterview und nicht die Selbstdarstellung beurteilten, das heißt, sie wussten nicht, wie lange die Bewerber in der vorhergehenden Aufgabenphase sich selbst dargestellt hatten. Wie ist es zu erklären, dass die Dauer der Selbstdarstellung ein relevanter Prädiktor für die Fremdeinschätzung des Bewerberverhaltens in einer späteren Aufgabe war? Hierfür gibt es zwei Erklärungsansätze, die sich nicht ausschließen, sondern eher ergänzen dürften. Zum einen ist $\mathrm{zu}$ vermuten, dass ein situationsübergreifendes Persönlichkeitsmerkmal (wie Selbstsicherheit) das Verhalten der Probanden in beiden Aufgaben beeinflusste. Zum anderen mag ein erfolgreiches Bewältigen der Selbstdarstellungsaufgabe einen positiven Effekt auf das Verhalten der Probanden in der nachfolgenden Aufgabe, dem Bewerbungsinterview, gehabt haben.

Die Ergebnisse dieser Studie unterstützen den Befund von Diemand und Schuler (1991), wonach die mit einem Fragebogen erhobene assertive Selbstdarstellung der wesentliche Prädiktor für das erfolgreiche Abschneiden in einem Assessment-Center war. Bescheidenheit und Zurückhaltung sind in einer Bewerbungssituation nicht angesagt - dieses Ergebnis wird auch durch die Meta-Analyse (mit 51 Studien) zum Zusammenhang zwischen Persönlichkeitsmerkmalen und dem Abschneiden in einem Assessment-Center von Scholz und Schuler (1993) unterstützt. Sie ermittelten, dass neben Intelligenz, sozialer Kompetenz und Leistungsmotivation die Persönlichkeitseigenschaften Dominanz und Selbstvertrauen mittelhoch mit dem Assessment-Center Gesamtergebnis korrelierten, während Verträglichkeit keinen oder sogar einen eher negativen Bezug zu den Bewertungen im $\mathrm{AC}$ aufwies.

Durch die beiden Variablen „Dauer der Selbstdarstellung“ und „Neutralität des Gesichts“ können immerhin 23\% der Gesamtvarianz des Fremdurteils als erfolgreich aufgeklärt werden, wobei die Dauer der Selbstdarstellung der wichtigste Prädiktor ist. Die Ergebnisse stützen somit die Funktionalität von professionellen Bewerbungstrainings, in denen Bewerbungskandidaten lernen, sich gut zu verkaufen (Hesse \& Schrader, 1994) und sich möglichst gut durchzusetzen (Kellner, 1998). Ob Personen, die sich entsprechend der in Tabelle 1 aufgeführten Selbstdarstellungsregeln verhalten und den Eindruck eines „Weicheies“ (Kellner, 1998) vermeiden, dann tatsächlich beruflich kompetenter und erfolgreicher sind als Personen, die über eine weniger selbstsichere Selbstdarstellung im Bewerbungsinterview verfügen, oder ob eine solche Selbstdarstellung eher eine Störvariable (Schuler, 1996) darstellt, kann aufgrund dieser Studie natürlich nicht vorhergesagt werden. Aufschlussreich sind in diesem Zusammenhang die kürzlich 
berichteten Ergebnisse der prognostischen Validierung eines Potenzialanalyseverfahrens bei Angestellten in der Kreditwirtschaft (Diemand \& Schuler, 1998). Der Zeitraum zwischen der Erhebung der Prädiktoren (darunter verschiedene Selbstdarstellungsvariablen) im Potenzialanalyseverfahren und der Erhebung der Kriterien (Beurteilung der Mitarbeiter durch aktuelle Vorgesetzte) betrug bei der Mehrzahl der Teilnehmer mindestens 2 Jahre. Es zeigte sich, dass Selbstdarstellungsvariablen zwar die spätere Potenzial- bzw. Karriereeinschätzung durch die Vorgesetzten beeinflussten; allerdings wiesen die Selbstdarstellungsvariablen keine Validität auf hinsichtlich der Beurteilung der aktuellen und spezifischen beruflichen Kompetenz der Mitarbeiter. Als Konsequenz aus diesen Forschungsergebnissen wäre im Sinne einer ,bewerberorientierten Gestaltung von Beurteilungssituationen" (Köchling \& Körner, 1996) zu fordern, diese so zu gestalten, dass nicht nur assertive Selbstdarsteller eine Chance bekommen.

Zwei methodische Einschränkungen der hier vorgestellten Studie seien abschließend genannt. Die Probanden hatten keine echten Interviewpartner, sie bekamen die Fragen und Instruktionen vom Tonband und antworteten in eine Videokamera (wobei sie sich vorstellen sollten, dass sie einer Bewerbungskommission gegenübersitzen würden). Es ist anzunehmen, dass sowohl die nonverbale Expressivität des Gesichts als auch die Länge der Selbstdarstellung durch die Anwesenheit von realen Kommunikationspartnern beeinflusst wird. Von daher wäre eine Replizierung der hier vorgestellten Studie in echten Bewerbungssituationen wünschenswert. Der zweite Punkt betrifft die Fremdeinschätzungen der Probanden, die von zwei weiblichen Beurteilern vorgenommen wurden. Es wäre zu überprüfen, ob die hier berichteten Zusammenhänge auch zu finden sind, wenn männliche Beurteiler den Erfolg der Probanden einschätzen.

\section{Literatur}

Bänninger-Huber, E. \& Salisch, M. von (1994). Die Untersuchung des mimischen Affektausdrucks in face-to-face-Interaktionen. Psychologische Rundschau, 45, 79-98.

Diemand, A. \& Schuler, H. (1991). Sozial erwünschtes Verhalten in eignungsdiagnostischen Situationen. In H. Schuler \& U. Funke (Hrsg.), Eignungsdiagnostik in Forschung und Praxis (Beiträge zur Organisationspsychologie, Band 10, S. 242-248). Stuttgart: Verlag für Angewandte Psychologie.

Diemand, A. \& Schuler, H. (1998). Wirksamkeit von Selbstdarstellungsvariablen im Rahmen der prognostischen Validierung eines Potentialanalyseverfahrens. Zeitschrift für Arbeits- und Organisationspsychologie, 42, 134-146.
Ekman, P. \& Friesen, W.V. (1969). The repertoire of nonverbal behavior: Categories, origins, usage and coding. Semiotica, 1, 49-98.

Ekman, P. \& Friesen, W. V. (1978). The facial action coding system. Palo Alto: Consulting Psychologist Press.

Fittkau, B. (1995). Kommunikation. In W. Sarges (Hrsg.), Management-Diagnostik (S.382-394). Göttingen: Hogrefe.

Graudenz, H. (1987). Eignungsuntersuchungen aus der Sicht der Bewerber. DGP Informationen, 38, 1-28. .

Graudenz, H. (1995). Sozialpsychologische Implikationen der Evaluation. In W. Sarges (Hrsg.), Management-Diagnostik (S. 862-868). Göttingen: Hogrefe.

Herbeck, M. B. (1994). Charisma und Maske. Die Expressivität von Mädchen und Jungen in der Pubertät. Unveröff. Diss., Universität Ulm.

Hesse, J. \& Schrader, H. C. (1994). Assessment-Center . Dashärteste Personalauswahlverfahren. Frankfurt/M.: Eichborn.

Hochschild, A. R. (1979). Emotion work, feeling rules, and social structure. American Journal of Sociology, 85, 551-575.

Jäger, R. S. (1995). Eignungsdiagnostik aus der Sicht der Kandidaten. In W. Sarges (Hrsg.), Management-Diagnostik (S. 102-108). Göttingen: Hogrefe.

Jochmann, W. (1984). Der implizite diagnostische Prozeß in der Personalberatung und seine aussagenlogische Formalisierung. Psychologie und Praxis - Zeitschrift für Arbeitsund Organisationspychologie, 28, 119-129.

Kellner, H. (1998). Das geheime Wissen der Personalchefs. Wie sie testen. Was sie suchen. Welche Persönlichkeitsmerkmale zum Erfolg führen. Frankfurt: Eichborn.

Köchling, A.C. \& Körner, S. (1996). Personalauswahl aus der Sicht der Betroffenen: Zur bewerberorientierten Gestaltung von Beurteilungssituationen. Zeitschrift für Arbeitsund Organisationspsychologie, 40, 22-37.

Scholz, G. \& Schuler, H. (1993). Das nomologische Netzwerk des Assessment Centers: eine Metaanalyse. Zeitschrift für Arbeits- und Organisationspsychologie, 37, 73-85.

Schuler, H. (1996). Psychologische Personalauswahl. Göttingen: Verlag für Angewandte Psychologie.

Traue, H.C. (1989). Gefühlsausdruck, Hemmung und Muskelspannung unter sozialem Streß. Göttingen: Hogrefe.

Wallbott, H. G. (1995). Ausdruck und Eindruck. In W. Sarges (Hrsg.), Management-Diagnostik (S. 394-400). Göttingen: Hogrefe.

Weber, H. \& Leger, S. (1993). Soziale Bewältigungsregeln (Memorandum Nr.21), Bamberg: Otto-Friedrich-Universität, Lehrstuhl Psychologie IV.

Wottawa, H. (1995). Formalisierung der Urteilsbildung. In W. Sarges (Hrsg.), Management-Diagnostik (S.785-789). Göttingen: Hogrefe.

Eingegangen: 30.05 .1998

Revision eingegangen: 10.02.1999

Priv.-Doz. Dr. Monika Sieverding

Freie Universität Berlin z.Zt. Gastprofessorin am Institut für Arbeits-, Organisations- und Gesundheitspsychologie, Habelschwerdter Allee 45, D-14195 Berlin. 